\title{
Planet Formation: Knowing the Progenitors and the Progeny
}

\author{
Kaitlin Kratter* \\ University of Colorado, Boulder \\ E-mail: kaitlin.kratterejila.colorado.edu
}

In this talk I provide an overview of the current theories of planet formation. I begin with a review of the processes that control the evolution of the protostellar and protoplanetary disks, setting the stage for planet formation. I focus on the core accretion model for planet formation, describing four basic phases: grain growth, planetesimal formation, envelope growth and migration and scattering. I describe how the Kepler mission has provided key observational evidence for this model of planet formation. These successes aside, there remain many open questions in planet formation theories. I briefly describe ongoing work on the importance of non-ideal MHD, stellar multiplicity, and disk chemistry.

Frank N. Bash Symposium 2013: New Horizons in Astronomy (BASH 2013)

October 6-8, 2013

Austin, Texas

${ }^{*}$ Speaker. 